\title{
Computing the Steady State Dynamics: The Chemical Graphs of Complex Reaction
}

Research Article

\section{Bilal Ali ${ }^{1 *}$, Sidra Jubair ${ }^{2 \dagger}$}

${ }^{1}$ School of mathematical sciences and Computing Technology, Central South University China.

${ }^{2}$ School of Mathematical Science Dalian, University of Technology, Dalian 116024, China

\begin{abstract}
In a thermodynamically isolated system, in order to obtain numerical approximations of the complex models, different model reduction techniques are applied to reduce the complex chemical reactions from high dimensional to low dimensional manifolds. These techniques not only reduce the system but also provide the complete description of reaction kinetics. These techniques include Quasi Steady State Approximations, Partial Equilibrium Technique, Intrinsic Low Dimensional Manifold Method, etc. Among all these techniques, Spectral Quasi Equilibrium Manifold Method is one of the most convenient way to find the initial approximations of slow invariant manifolds. Initial invariant grids completely describe the slow invariant manifolds which are obtained from the dissipative system. This method is applicable for high dimensional complex chemical reactions.

MSC: $\quad$ 74Gxx; 37Mxx

Keywords: Steady state; Chemical Reaction; Invariant manifold; Nodes and Trees
\end{abstract}

\section{Introduction}

In chemical kinetics, we control the speed of chemical reaction by different conditions. It provides the information about chemical reaction mechanism and transition state. Besides this, if pressure temperature medium concentration is known we can measure the rate of change of reactants and products with respect to time. Chemical kinetics covers three features of a chemical reaction. In rate of reaction temperature, pressure, concentration, catalyst, and solvent are controlled. Simply chemical kinetics is the speed of chemical reaction (Anatol., 2005). Thermodynamics deals with the principle of conservation of energy. It mainly circulates around the system, surrounding and boundary. Boundary separate the system and surrounding which may be real or imaginary. The system may be

\footnotetext{
*E-mail: bilal.maths_csu@yahoo.com

${ }^{\dagger}$ E-mail: Sidra.jubair@gmail.com
} 
open, isolated and closed. Chemical kinetics challenges and exploits area in which chemistry, mathematics, physics, and chemical engineering are covered. Basically, chemical kinetics is a complex combination of different fields.

Chemistry wants math and arithmetic craving demonstrating for its arrangement. The connection among Math's and chemistry ascends in the eighteenth - twentieth hundreds of years. Lavoisier and Dalton was known as pioneers of substance stoichiometry. There are various parts of chemistry and chemical energy is the hereditary piece of science. It has huge applications in the logical field. From another side, the creator of matrices theory "Arthur Cayley' applied the theory of chemical isomers in the 1860s-1870s, then in mid of 19th -century various laws of chemical kinetics were developed some of them are

- The mass-action law by Guldberg and Waage.

- The "normal classification of reactions" by van't Hoff.

- The quasi-steady-state principle by Chapman and Bodenstein.

- Theory of chain reactions by Semenov and Hinshelwood.

Every one of these above standards depend on a strong numerical establishment of the arithmetical and differential condition. In 1950-60s the very fast reactions were studied by relaxation technique (Eigen). (book Chemical Engineering)

The rate-determining step is frequently not self-evident when a reaction mechanism has several steps of comparable rates. However, in some steps there is an Intermediate. An intermediate is a species that is neither one of the reactants, nor one of the products. The steady-state approximation is actually used to find the Rate Law. The Strategy depends on the approximation that one intermediate in the reaction mechanism is burned-through as fast as it is created. The concentration of the reaction remains the same in a desired duration. At the point when a response includes at least one or more intermediates, the concentration of one of the intermediates remains constant at some stage of the reaction. Thus, the system has reached a steady-state. The convergence of one of the intermediates, varies with time.

Gibbs rule was introduced by Josiah Willard Gibbs from 1875-1878. The general form of the rule is given as,

$$
F=C-P+2
$$

Equivalently, Gibbs Rule can also be written as

$$
N_{k c}=N_{c}-\operatorname{Rank}(B)
$$

where $N_{k c}$ represents the number of key components (Degree of Freedom), $N_{c}$ is the total number of species involved in a chemical reaction and $\operatorname{Rank}(B)$ is the rank of balancing matrix. 


\section{Model Reaction Scheme}

\subsection{Lyapunov Function}

The Lyapunov function is a scalar function that is used to determine the constancy of an equilibrium point of ODE's. The Lyapunov function is symbolized by $G$ and can be characterized for a closed system as

$$
G=\sum_{j=1}^{n} c_{j}\left[\ln \left(c_{j} / c_{j}^{e q}\right)-1\right]
$$

\subsection{Quasi-Equilibrium Manifold}

Mathematically, QEM can be defined as:

$$
\left\{\begin{array}{l}
G \rightarrow \min \\
\left(m_{i}, c\right)=\xi_{i} \quad \forall_{i}=1, \ldots, k \\
B C=\text { const }
\end{array}\right.
$$

Here $m_{i}$ are slow variables and $\xi_{i}$ are the reduced description having $c_{i}$ elements. The general idea of QEM is represented as in figure given below:

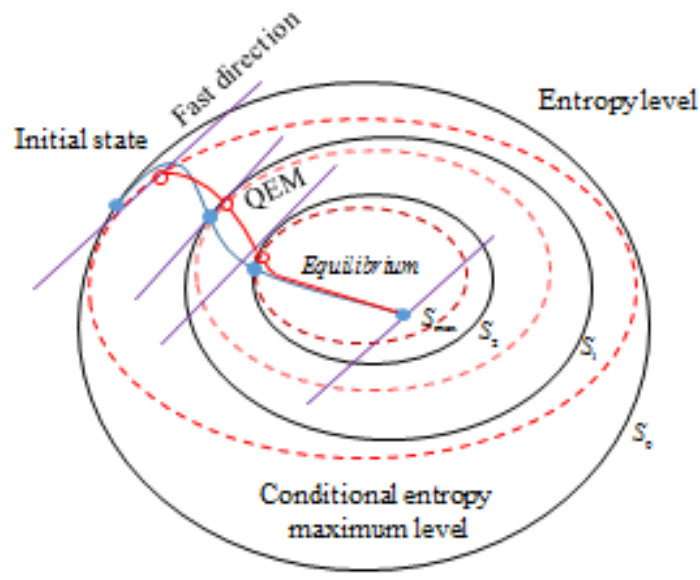

Figure (1): blue line represents initial state while the red line denotes the QEM. (Mahdi et, al)

\subsection{Spectral Quasi-Equilibrium Manifold}

In SQEM, left slowest eigenvector corresponding to the Jacobian matrix is selected. So, the solution obtained by selecting left slowest eigenvector of the Jacobian matrix corresponding to the smallest absolute eigenvalue is called Spectral Quasi-Equilibrium Manifold. (E. Chiavazzo, A.N Gorban, and I.V Karlin). 


\subsection{D Quasi-Equilibrium Grid Construction Method}

Since our interest is to find 1D Quasi-Equilibrium Manifold. For this, we select a node $c_{0}$ which is the equilibrium point related to that manifold which we have to assume. We will move towards further node points $c_{1}, c_{2}, c_{3} \ldots . . c_{n}$ lying on this manifold. Now, in order to find next node $c_{1}$, following formula will be used. i.e.

$$
\mathrm{c}_{1}=c_{0}+\hat{\delta} c_{0}
$$

By using above, we can find the next node up to $c_{n+1}$ of Quasi-Equilibrium Manifold if $c_{n}$ is known. Now the shift vector $\delta c_{n}$ used in equation (3.3) can be defined a

$\delta c_{n}=\sum_{i}^{n} \alpha_{i} p_{i}$

Here, $\left\{p_{i}\right\}$ is the $n-1$ dimensional null space basis of molecular balancing matrix $B$ and the unknown variables $\alpha_{i}$ 's can be calculated as

$$
\begin{aligned}
& \sum_{i=1}^{k}\left(t_{j}, p_{i}\right) \alpha_{i}=-\left(t_{j}, \nabla G\left(c_{i}\right)\right) \quad \forall j=1,2 \ldots, k-1 \\
& \sum_{i=1}^{k} \alpha_{i}^{2}=\varepsilon
\end{aligned}
$$

Where $\varepsilon$ is a small parameter.

Now $E$ matrix can be achieved by combining slowest left Eigenvector $m$ and the balancing matrix $B$ as follows:

$$
\mathrm{E}=\left[\begin{array}{l}
m \\
B
\end{array}\right]
$$

Now consider that the node point $c_{x}$ is very near to QEM and $\delta c_{x}$ be very lesser such that $\nabla G$ which is the gradient of $G$ can be estimated to $1^{\text {st }}$ order as:

$$
\nabla G\left(c_{x+1}\right) \cong \nabla G\left(c_{x}\right)+H\left(c_{x}\right) \delta c_{x}
$$

Here, $H\left(c_{x}\right)$ symbolizes the enthalpy which is the $2^{\text {nd }}$ derivative of the Lyapunov function $G$ and is equal to

$$
H\left(c_{x}\right)=\left[\frac{\partial^{2} G\left(c_{x}\right)}{\partial x_{i} \partial y_{j}}\right]
$$

By substituting equation (3.4) and Equation (3.8) in equation (3.7), we will get the following:

$$
\sum_{i=1}^{k}\left(t_{j}, p_{i}\right) \alpha_{i}=-\left(t_{j}, \nabla G\left(c_{x}\right)\right) \quad \forall j=1,2, \ldots k-1
$$

Note that the right-hand side of the above equation will be zero if the node points $c_{x}$ belong to QEM. 
Since, we know that in order to find out the solution of $n$ number of unknown variables, the system of ODE's need $n$ number of equations. But here in equation (3.11), we have $k$ unknowns and $k-1$ equations. Therefore, we don't have proper information about grid spacing. To overcome this problem, we fix the given space as:

$$
\left\{\begin{array}{l}
\sum_{i=1}^{k}\left(t_{j}, p_{i}\right) \alpha_{i}=-\left(t_{j}, \nabla G\left(c_{x}\right)\right) \quad \forall j=1,2, \ldots k-1 \\
\left\|\delta c_{x}\right\|=\varepsilon
\end{array}\right.
$$

Here, $\left\|\delta c_{x}\right\|$ represents the Euclidian norm of the vector $\delta c_{x}$. Also $\varepsilon$ is a small parameter. By choosing a small value of this parameter, the accuracy will be clear.

Now by choosing $\left\{p_{i}\right\}$ as orthonormal basis of the above space, the above-transformed system (3.12) can be written as:

$$
\left\{\begin{array}{l}
\sum_{i=1}^{k}\left(t_{j}, p_{i}\right) \alpha_{i}=-\left(t_{j}, \nabla G\left(c_{x}\right)\right) \quad \forall j=1,2, \ldots k-1 \\
\sum_{i=1}^{k} \alpha_{i}^{2}=\varepsilon^{2}
\end{array}\right.
$$

The system (3.13) is the required model. Node points finding from (3.13) are known as QEG. By choosing these node points, we will get all the desired forward and backward node points in both directions.

Note that in QEG, the equilibrium point is chosen as the initial point for the construction. i.e. $c_{e q}=c_{o}$

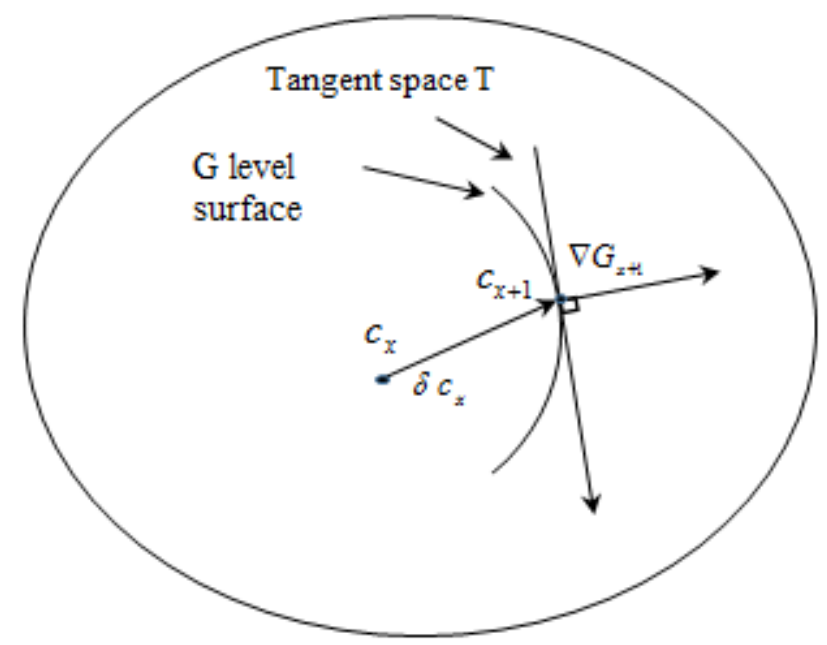

Figure 2: General idea to proceed from one node point $c_{x}$ to next node point $c_{x+1}$.(Chiavazzo., I.V. Karlin (2007)).

We now distinguish those trajectories that move along the fast and slow direction. 


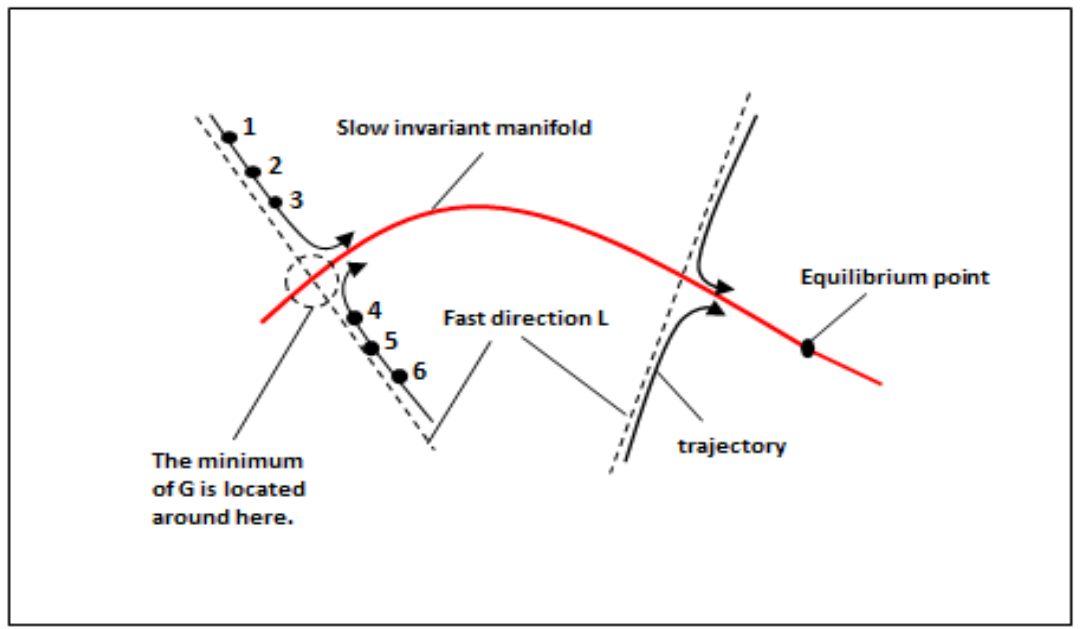

Figure 9: Fast and slow trajectories moving towards the equilibrium point.

(Chiavazzo, E., Gorban, A. N., and Karlin, I. V., 2007

\section{Working Example:}

Further explain the above techniques we consider an example, let us suppose the general parallel adsorption mechanism of two steps reversible

$$
\begin{aligned}
& A+Z \underset{k_{1}^{-}}{\stackrel{k_{1}^{+}=1}{\rightleftharpoons}} A Z \\
& A Z+B \underset{k_{2}^{-}}{\stackrel{k_{2}^{+}=0.5}{\rightleftharpoons}} A B+Z
\end{aligned}
$$

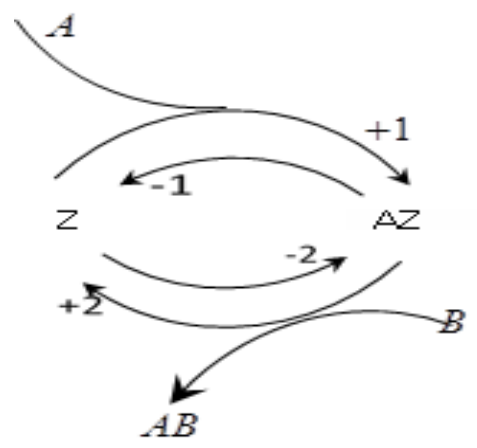

Figure 3: In this figure $\mathrm{A}$ and $\mathrm{B}$ are reactants and $\mathrm{AB}$ is a product. $\mathrm{Z}$ and $\mathrm{AZ}$ are intermediates.

\subsection{Trees and Nodes:}

Since it is two-step reversible chemical reaction. So, by Cayley's formula, the total number of trees will be four. While total forward and reverse trees will be equal i.e. two. Also, the total number of combined trees will be zero in this case. 


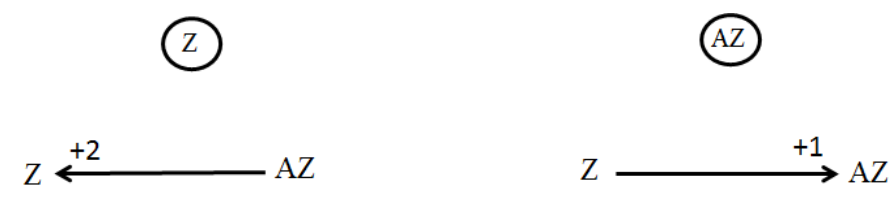

Figure 11: represents the forward spanning trees of the node $\mathrm{Z}$ and $\mathrm{AZ}$.

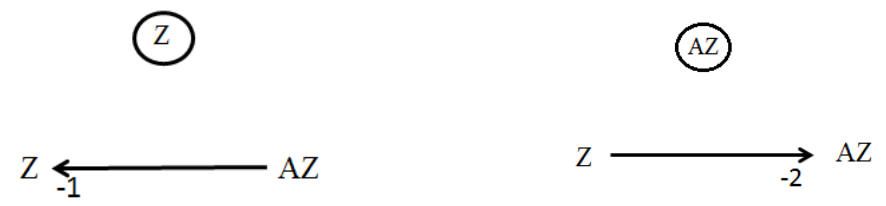

Figure 4: represents the reverse spanning trees of the node $\mathrm{Z}$ and $\mathrm{AZ}$.

Now, by finding its edge weight, we have forward reaction weights given as

$$
\begin{aligned}
& W_{1}^{+}=\frac{\gamma_{1}^{+}}{\theta_{Z}}=\frac{K_{1}^{+} C_{A} \theta_{Z}}{\theta_{Z}}=K_{1}^{+} C_{A} \\
& W_{2}^{+}=\frac{\gamma_{2}^{+}}{\theta_{A Z}}=\frac{K_{2}^{+} C_{B} \theta_{A Z}}{\theta_{A Z}}=K_{2}^{+} C_{B}
\end{aligned}
$$

And reverse reaction weights will be equal to

$$
\begin{aligned}
& W_{1}^{-}=\frac{\gamma_{1}^{-}}{\theta_{A Z}}=\frac{K_{1}^{-} \theta_{A Z}}{\theta_{A Z}}=K_{1}^{-} \\
& W_{2}^{-}=\frac{\gamma_{2}^{-}}{\theta_{Z}}=\frac{K_{2}^{-} C_{A B} \theta_{Z}}{\theta_{Z}}=K_{2}^{-} C_{A B}
\end{aligned}
$$

Now by finding the weight of the spanning trees of node $Z$ and $A Z$, we will get

$$
\begin{aligned}
& W_{Z}=w_{2}^{+}+w_{1}^{-} \\
& W_{A Z}=w_{1}^{+}+w_{2}^{-}
\end{aligned}
$$

Thus the total weight of spanning trees of whole graph will be

$$
W=W_{Z}+W_{A Z}
$$

\subsection{Mathematical Modeling}

In this two-step chemical reaction, five chemical components are involved ( $\mathrm{A}, \mathrm{Z}, \mathrm{AZ}, \mathrm{B}, \mathrm{AB})$ which are formed by three chemical elements (A, $\mathrm{Z}$ and $\mathrm{B})$. Besides this, we also define initial parameters i.e. 


$$
\begin{aligned}
& A=c_{1}, Z=c_{2}, A Z=c_{3}, B=c_{4}, A B=c_{5} \\
& c_{1}^{e q}=0.5, c_{2}^{e q}=0.1, c_{3}^{e q}=0.1, c_{4}^{e q}=0.4, c_{5}^{e q}=0.1
\end{aligned}
$$

Since we have a 5- dimensional matrices so we transform it into 2-dimensional metrics by defining a valid balancing equation given by the law of conservation of atoms.

$$
B=\left[\begin{array}{lllll}
1 & 0 & 1 & 0 & 1 \\
0 & 1 & 1 & 0 & 0 \\
0 & 0 & 0 & 1 & 1
\end{array}\right]
$$

Then stoichiometric vectors are calculated as:

$$
\begin{aligned}
& \gamma_{1}=(-1,-1,1,0,0) \\
& \gamma_{2}=(0,1,-1,-1,1)
\end{aligned}
$$

And the stoichiometric matrix $\gamma$ becomes:

$$
\gamma=\left[\begin{array}{rrrrr}
-1 & -1 & 1 & 0 & 0 \\
0 & 1 & -1 & -1 & 1
\end{array}\right]
$$

Now for kinetic equation, we have the following form

$$
c^{\bullet}=\sum_{i=1}^{2} \gamma_{i} R_{i}
$$

i.e., the product of stoichiometric vectors ' $\gamma_{i}$ ' and reaction rate' $R_{i}$ '. So by expanding the above system, we get:

$$
c^{\bullet}=\gamma_{1} R_{1}+\gamma_{2} R_{2}
$$

By substituting these values and then simplifying, we seek following five kinetic equations:

$$
\begin{aligned}
c_{1}^{\bullet} & =-k_{1}^{+} c_{1} c_{2}+k_{1}^{-} c_{3} \\
c_{2}^{\bullet} & =-k_{1}^{+} c_{1} c_{2}+k_{1}^{-} c_{3}+k_{2}^{+} c_{3} c_{4}-k_{2}^{-} c_{5} c_{2} \\
1 \quad c_{3}^{\bullet} & =+k_{1}^{+} c_{1} c_{2}-k_{1}^{-} c_{3}-k_{2}^{+} c_{3} c_{4}+k_{2}^{-} c_{5} c_{2} \\
c_{4}^{\bullet} & =-k_{2}^{+} c_{3} c_{4}+k_{2}^{-} c_{5} c_{2} \\
c_{5}^{\bullet} & =+k_{2}^{+} c_{3} c_{4}-k_{2}^{-} c_{5} c_{2}
\end{aligned}
$$

Here $c_{i}^{\bullet}$ are derivative with respect to time and all $c_{i}$ 's are variables and

$$
\begin{array}{ll}
k_{1}^{-}=0.500 & k_{1}^{+}=1.000 \\
k_{2}^{-}=2.000 & k_{2}^{+}=0.500
\end{array}
$$


We can write all equations in matrix form as.

$$
c^{\bullet}=\left[\begin{array}{c}
c_{1}^{\cdot} \\
c_{2}^{\bullet} \\
c_{3}^{\cdot} \\
c_{4}^{\cdot} \\
c_{5}^{\bullet}
\end{array}\right]=\left[\begin{array}{l}
-k_{1}^{+} c_{1} c_{2}+k_{1}^{-} c_{3} \\
-k_{1}^{+} c_{1} c_{2}+k_{1}^{-} c_{3}+k_{2}^{+} c_{3} c_{4}-k_{2}^{-} c_{5} c_{2} \\
+k_{1}^{+} c_{1} c_{2}-k_{1}^{-} c_{3}-k_{2}^{+} c_{3} c_{4}+k_{2}^{-} c_{5} c_{2} \\
-k_{2}^{+} c_{3} c_{4}+k_{2}^{-} c_{5} c_{2} \\
+k_{2}^{+} c_{3} c_{4}-k_{2}^{-} c_{5} c_{2}
\end{array}\right]
$$

Since the molecular or balancing matrix is the balance of $A, Z$ and $B$. So we can write (26) as:

$$
B=\left[\begin{array}{ccccc}
1 & 0 & 1 & 0 & 1 \\
0 & 1 & 1 & 0 & 0 \\
0 & 0 & 0 & 1 & 1
\end{array}\right] \cdot\left[\begin{array}{l}
b_{A} \\
b_{Z} \\
b_{B}
\end{array}\right]
$$

Also, each species of molecular matrix satisfies the law of conservation of mass, i.e.,

$$
B c=\text { Constant }
$$

By substituting values in (28), we get

$$
\left[\begin{array}{lllll}
1 & 0 & 1 & 0 & 1 \\
0 & 1 & 1 & 0 & 0 \\
0 & 0 & 0 & 1 & 1
\end{array}\right] \cdot\left[\begin{array}{l}
c_{1} \\
c_{2} \\
c_{3} \\
c_{4} \\
c_{5}
\end{array}\right]=\left[\begin{array}{l}
b_{A} \\
b_{Z} \\
b_{B}
\end{array}\right]
$$

By simplifying (29) and by putting the values of all parameters i.e.

$$
c_{1}^{e q}=0.5, c_{2}^{e q}=0.1, c_{3}^{e q}=0.1, c_{4}^{e q}=0.4, c_{5}^{e q}=0.1
$$

The equation (27) becomes:

$$
\left[\begin{array}{l}
b_{A} \\
b_{Z} \\
b_{B}
\end{array}\right]=\left[\begin{array}{l}
0.7 \\
0.2 \\
0.5
\end{array}\right]
$$

So we can write it as:

$$
\begin{aligned}
c_{1}+c_{3}+c_{5} & =b_{A}=0.7 \\
c_{2}+c_{3} & =b_{Z}=0.2 \\
c_{4}+c_{5} & =b_{B}=0.5
\end{aligned}
$$


Now By fixing $c_{1}$ and $c_{4}$ and by putting all values we get the reduced form of the kinetic equation and it can be written in matrix form as:

$$
F=\left[\begin{array}{c}
-c_{1}^{2}-0.5 c_{1}+0.5 c_{4}+c_{1} c_{4}+0.1 \\
1.5 c_{4}^{2}-1.1 c_{4}-1.5 c_{1} c_{4}+c_{1}
\end{array}\right]
$$

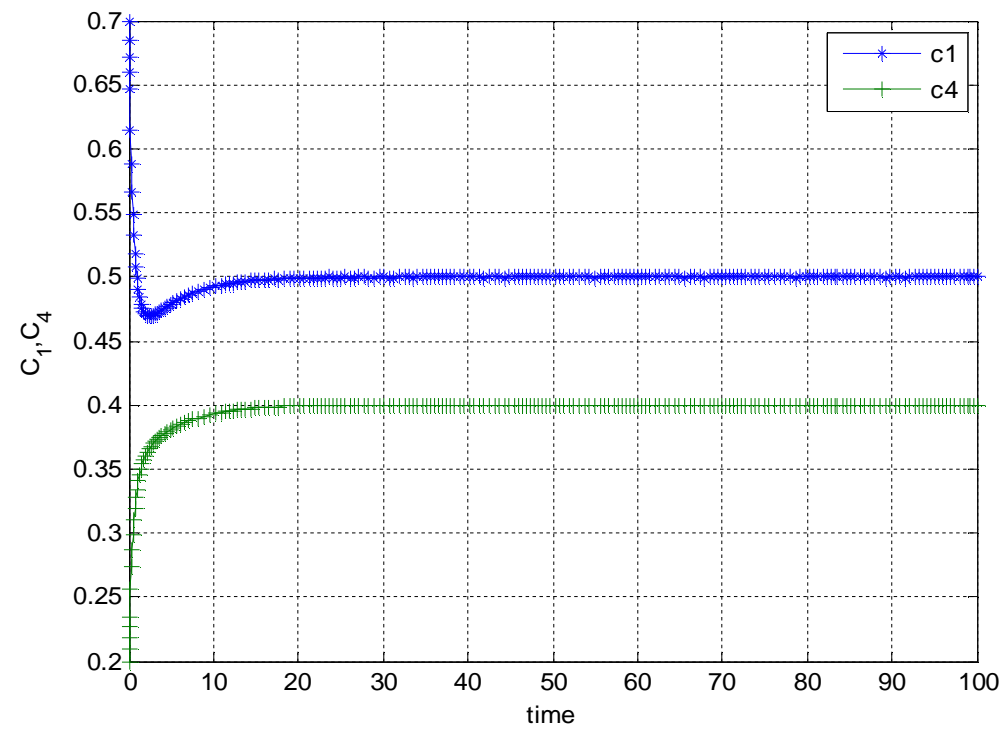

Figure 5: Quasi-steady-state assumption in reduced equations of reaction in terms of $c_{1}$ and $c_{4}$.

\subsection{Implementing 1D-SQEG Algorithm}

Now we will apply SQEGA method to our problem i.e. Consider the kinetic equations from equation (4.22), we have

$$
J(c)=c_{i}^{\cdot}=\left[\begin{array}{l}
-k_{1}^{+} c_{1} c_{2}+k_{1}^{-} c_{3} \\
-k_{1}^{+} c_{1} c_{2}+k_{1}^{-} c_{3}+k_{2}^{+} c_{3} c_{4}-k_{2}^{-} c_{5} c_{2} \\
+k_{1}^{+} c_{1} c_{2}-k_{1}^{-} c_{3}-k_{2}^{+} c_{3} c_{4}+k_{2}^{-} c_{5} c_{2} \\
-k_{2}^{+} c_{3} c_{4}+k_{2}^{-} c_{5} c_{2} \\
+k_{2}^{+} c_{3} c_{4}-k_{2}^{-} c_{5} c_{2}
\end{array}\right]
$$

Now measuring the Jacobian of above matrix by using mat lab, we get:

$$
J(c)=\left[\begin{array}{rrrcc}
-0.1000 & -0.5000 & 0.5000 & 0 & 0 \\
-0.1000 & -0.7000 & 0.7000 & 0.0500 & -0.2000 \\
0.1000 & 0.7000 & -1.0000 & 0 & 0.2000 \\
0 & 0.2000 & -0.2000 & -0.0500 & 0.2000 \\
0 & -0.2000 & 0.2000 & 0.0500 & -0.2000
\end{array}\right]
$$


Now we can find Eigen values $|J-\lambda I|=0$ and Eigen vectors $(J-\lambda I) X=0$ easily. But our task is to find the left slowest Eigenvector w.r.t Eigenvalues in order to implement the SQEM technique to this problem. For this, we measure its first left Eigenvalues of the above matrix (4.36) w.r.t mat lab, we have:

$$
\lambda=\left[\begin{array}{lllll}
-1.6894 & 0 & 0 & 0 & 0 \\
0 & 0.2219 & 0 & 0 & 0 \\
0 & 0 & -0.1387 & 0 & 0 \\
0 & 0 & 0 & 0.0000 & 0 \\
0 & 0 & 0 & 0 & 0.0000
\end{array}\right]
$$

And its corresponding Eigenvectors by using mat lab:

$$
\vec{W}=\left[\begin{array}{rrrrr}
-0.0866 & -0.1573 & 0.2272 & -0.2662 & -0.2662 \\
-0.6212 & 0.1041 & 0.6279 & 0.2662 & 0.2662 \\
0.7554 & 0.2959 & 0.5400 & -0.0000 & -0.0000 \\
0.0247 & -0.2893 & -0.0677 & 0.5731 & 0.5731 \\
-0.1882 & 0.8906 & -0.5078 & 0.3069 & 0.3069
\end{array}\right]
$$

Now for left slowest Eigenvector, we have seen that $/ \lambda_{3} /</ \lambda_{2} /</ \lambda_{1} /$. Hence left slowest Eigenvector corresponding to $\lambda_{3}$ will be:

$$
L s=\left[\begin{array}{lllll}
0.2272 & 0.6279 & 0.5400 & -0.0677 & -0.5078
\end{array}\right]
$$

Now defining the Lyapunov function as:

$$
G=\sum_{j=1}^{n} c_{j}\left[\ln \left(c_{j} / c_{j}^{e q}\right)-1\right]
$$

Also, Gradient of Lyapunov function $\nabla G$ and its $2^{\text {nd }}$ derivative $H$ will become:

$$
\begin{aligned}
& \nabla G=\left[\begin{array}{c}
\log \left(c_{1}\right)-\log \left(c_{1}^{e q}\right) \\
\log \left(c_{2}\right)-\log \left(c_{2}^{e q}\right) \\
\log \left(c_{3}\right)-\log \left(c_{3}^{e q}\right) \\
\log \left(c_{4}\right)-\log \left(c_{4}^{e q}\right) \\
\log \left(c_{5}\right)-\log \left(c_{5}^{e q}\right)
\end{array}\right] \\
& H=\left[\begin{array}{ccccc}
1 / c_{1} & 0 & 0 & 0 & 0 \\
0 & 1 / c_{1} & 0 & 0 & 0 \\
0 & 0 & 1 / c_{3} & 0 & 0 \\
0 & 0 & 0 & 1 / c_{4} & 0 \\
0 & 0 & 0 & 0 & 1 / c_{5}
\end{array}\right]
\end{aligned}
$$


Now after that, we measure the next node points. In order to find the node points, we have following formula:

$$
c_{n+1}=c_{n}+\delta c_{n}
$$

Where,

$$
\delta c_{n}=\sum_{i}^{n} \alpha_{i} p_{i}
$$

Here in equation (43), $\alpha_{i}$ are the unknown variables which we have to find. To find the $\alpha_{i}$ 's, we have the system

$$
\begin{aligned}
& \sum_{i=1}^{2}\left(t_{j}, p_{i}\right) \alpha_{i}=-\left(t_{j}, \nabla G\left(c_{i}\right)\right) \quad \forall i=1,2 \\
& \sum_{i=1}^{2} \alpha_{i}^{2}=0.001
\end{aligned}
$$

$p_{i}$ represent the null basis of the balancing matrix i.e.

$$
p_{i}=\operatorname{null}(B)
$$

Calculating the null basis $p_{i}$ using mat lab software package, we have:

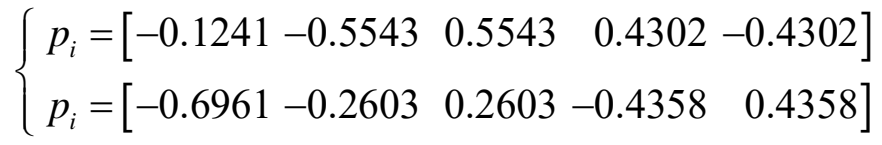

Also $t_{i}$ is the null basis of matrix $E$, i.e.

$$
t_{i}=\operatorname{null}(E)
$$

Where

$$
E=\left[\begin{array}{l}
L s \\
B
\end{array}\right]
$$

By putting values, we have:

$$
E=\left[\begin{array}{lllll}
0.2272 & 0.6279 & 0.5400 & -0.0677 & -0.5078 \\
1.0000 & 0 & 1.0000 & 0 & 1.0000 \\
0 & 1.0000 & 1.0000 & 0 & 0 \\
0 & 0 & 0 & 1.0000 & 1.0000
\end{array}\right]
$$

And,

$$
t=\left[\begin{array}{lllll}
0.3198 & 0.6058 & -0.6058 & -0.2861 & 0.2861
\end{array}\right]
$$

Now by putting values of $t_{i}$ and $p_{i}$, we get 


$$
\begin{aligned}
& \alpha_{1}=0.007519 \Rightarrow \alpha_{2}=-0.03072 \\
& \alpha_{1}=-0.007519 \Rightarrow \alpha_{2}=0.03072
\end{aligned}
$$

Now in order to find next point,

$$
c_{1}=c_{0}+\delta c_{0}
$$

By using $1^{\text {st }}$ value of $\alpha_{1}, \alpha_{2}$ and $p_{i}$ in equation (43), we get the value of $\delta c_{0}$ that is equal to

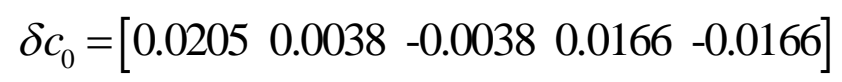

Substituting the values of $c_{0}$ and $\delta c_{0}$ in equation (51), we will acquire the next forward node point $c_{1}$. Similarly, we repeat this whole method again and get all the possible forward node points $c_{2}, c_{3}, \ldots, c_{n}$. By plotting all node points, we will get 1-D approximate SQEM solution in the forward direction.

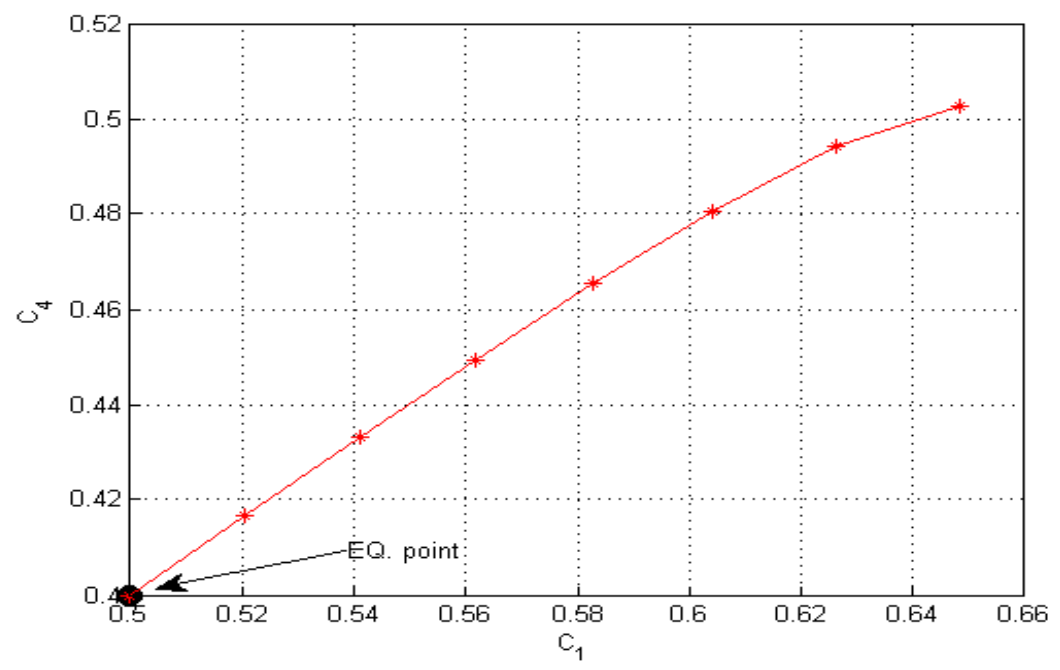

Figure 6: The above figure shows the initial approximation of SQEM solution in the forward direction.

Similarly, by using $2^{\text {nd }}$ value of $\alpha_{1} \cdot \alpha_{2}$ and $p_{i}$ in equation (4.45), we get the value of $\delta c_{0}$ that is equal to

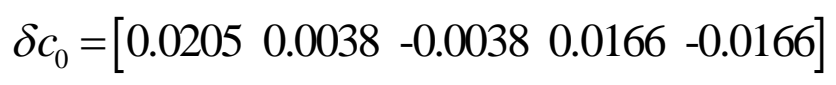

Similarly, we repeat this whole method again to get all the possible backward node points $c_{2}, c_{3}, \ldots ., c_{n}$. By plotting all those node points, we will get 1-D approximate SQEM solution in reverse direction. 


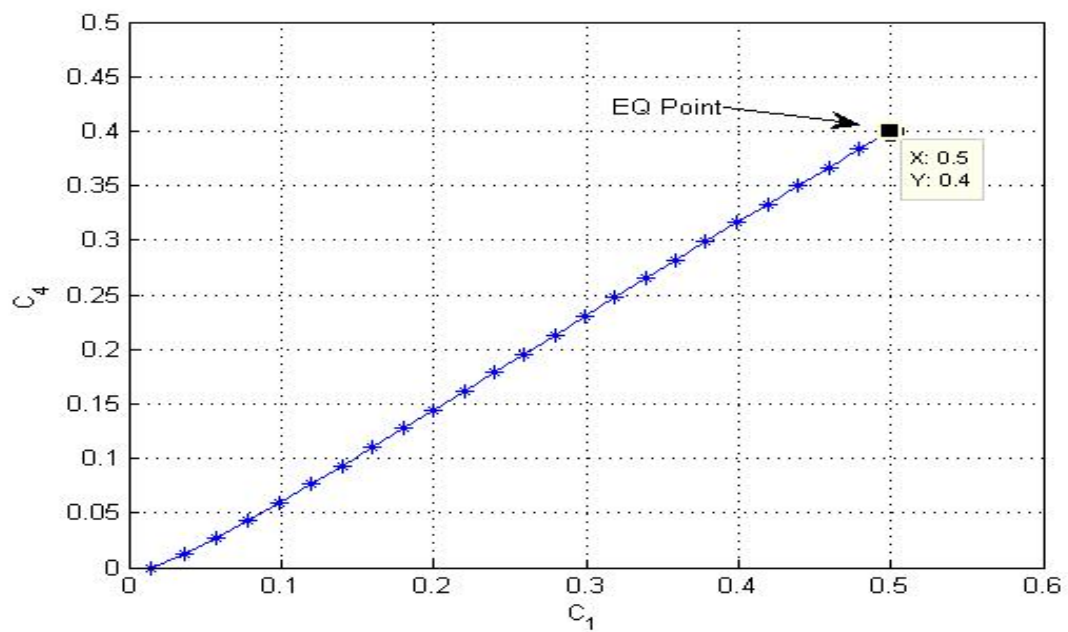

Figure 7: The above figure shows the initial approximation of SQEM solution in the backward direction.

Combining all forward and backward node points with equilibrium points. We will get:

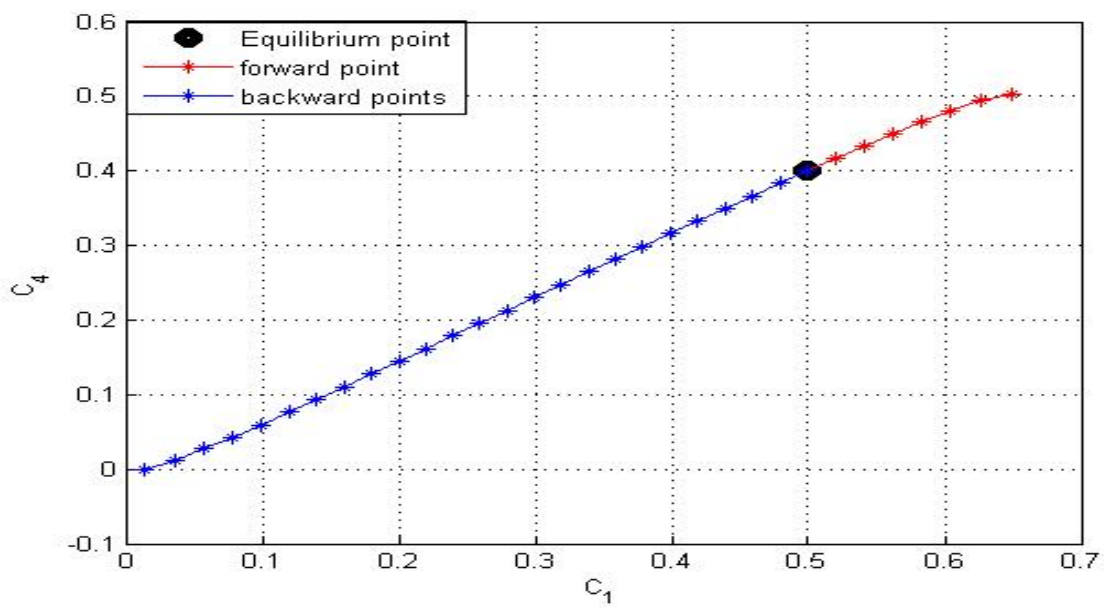

Figure 8: The above figure shows the initial approximation of SQEM solution in both forward and backward direction.

Now we will define the solution trajectories of the above-approximated solution of the SQEM solution: 


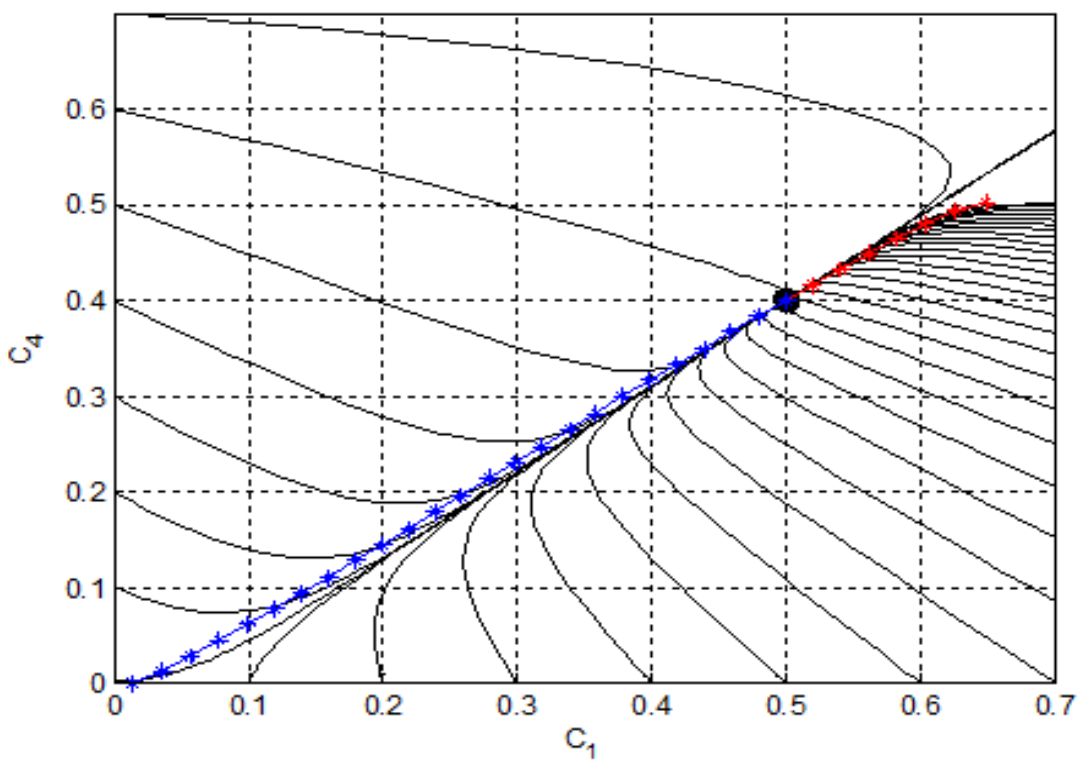

Figure 9: represents the approximated solution of SQEM with solution trajectories lies nearer to the equilibrium point.

(a)

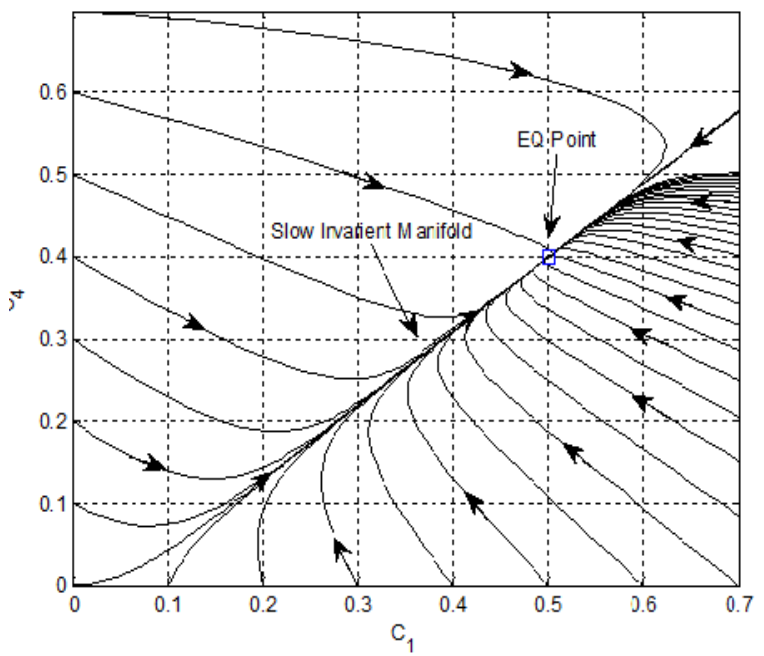

(b)

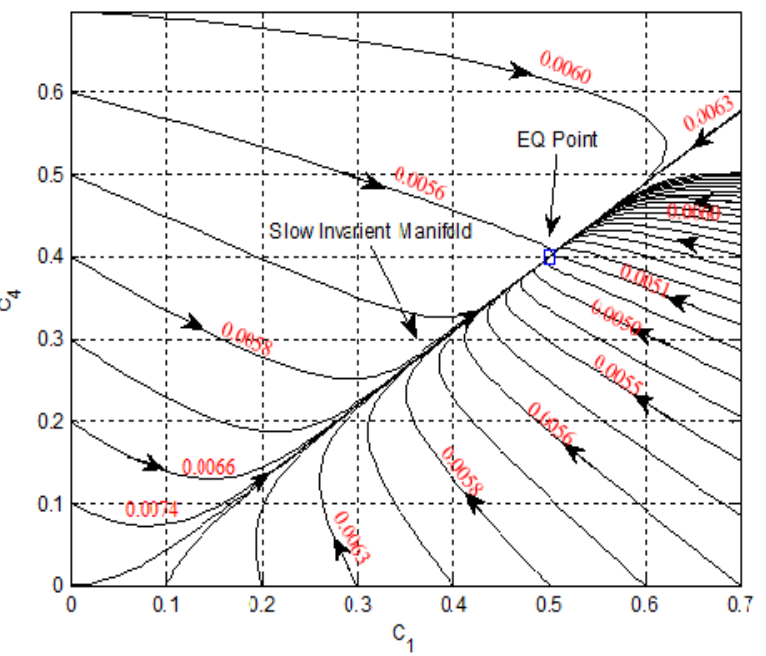

Figure 10 (a): shows the direction of trajectories moving towards equilibrium point and (b) shows the time taken by each trajectory to reach its equilibrium point

\section{Conclusion}

In this work two main goals are achieved, firstly the concept of trees and nodes of the chemical graph is presented with a suitable example by using Cayley's formula. Secondly, we have found the weights of spanning trees. After that, the graphical representation of computational methods (SQEM) is discussed in detailed to find the initial approximation for the slow invariant manifold of complex chemical reaction. Also, we have reduced the high dimensional problem to low dimensional by using Gibbs Rule and implement SQEM method to find 1-Dimensional Spectral Quasi-Equilibrium Manifold (SQEM) solution. 


\section{Acknowledgements}

Authors warmly thank the learned reviewers for their constructive suggestions.

\section{Competing Interests}

Authors declare that they have no competing interest.

\section{References}

[1] Waage, C. M., and Goldberg. Studies Concerning Affinity Forhandlinger: Videnskabs - Selskabet I Christinia (Norwegian academy of science and letters), 1864.

[2] Zumdahl., and S. Steven. Chemical Principles. 6th Ed. Boston: Houghton Mifflin, 2009.

[3] Laidler. K.J. Chemical Kinetics. 3rd Ed., Harper and Row. 277, 1987.

[4] Ansumali, S., and I.V. Karlin, Single relaxation time model for entropic Lattice Boltzmann methods. Phys. E. 65 (2002), 1-9.

[5] Denis Constales., Gregory S. Yablonsky, Dagmar R, D’hooge, Joris W. Thybaut, Guy B. Marin, Advanced Data Analysis and Modelling in Chemical Engineering, 2017.

[6] Chiavazzo, E., Gorban, A. N., and Karlin, I. V.. Comparison of Invariant Manifolds for Model Reduction in Chemical Kinetics. COMMUNICATIONS IN COMPUTATIONAL PHYSICS. 2 (2007), 964 - 992.

[7] Shahzad, M., Arif, H., Gulistan, M., and Sajid, M., Initially Approximated Quasi Equilibrium Manifold. Journal of the Chemical Society of Pakistan. 37 (2) (2015).

[8] Gorban, A. N., Karlin, I. V., and Zinovyev, A. Y., Constructive methods of invariant manifolds for kinetic problems. Physics Reports. 396 (2004), 197- 403.

[9] Kooshkbaghi, M., Frouzakis, C. E., Boulouchos, K., and Karlin, I. V., Spectral Quasi-Equilibrium Manifold for Chemical Kinetics. J. Phys. Chem. 120 (2016), 3406 -3413.

[10] Davis, M. J., and Skodje, R. T., Geometric investigation of low-dimensional manifolds in systems approaching equilibrium. J. Chem. Phys. 111(1999), 1-17.

[11] Tomlin, A. S., Turanyi, T., and Pilling, M. J., Mathematical tools for the construction, investigation, and reduction of combustion mechanisms. Comprehensive chemical kinetics. 35 (1997), 293 - 438.

[12] Chiavazzo, E., 2009. Invariant Manifold and Lattice Boltzmann Method for Combustion (Doctoral dissertation, ETH Zurich, Dipl.-Ing. University of Naples, Federico II).

[13] Gorban, A. N., and Shahzad, M., The Michaelis-Menten-Stueckelberg Theorem. Entropy. 13 (2011), 966-1019.

[14] Dorschner, D., Frapolli, N., Chikatamarla, S. S., and Karlin, I. V., Grid refinement for entropic lattice Boltzmann models. PHYSICS REVIEW E. 94 (2016), 053311-14.

[15] Gorban, A. N., Karlin, I. V., and Zinovyev, A. Y., Invariant grids for reaction kinetics. Physics A: Statistical Mechanism and its Applications. 333 (2004), 106 -154.

[16] Gorban, A. N., and Karlin, I. V., Method of invariant manifold for chemical kinetics. Cond-mat. statmech. 020723 (2002), 1- 42.

[17] Petzold, L., and Zhu, W., Model Reduction for Chemical Kinetics: An Optimization Approach. AIChE Journal. 45 (1999), 869 - 886.

[18] Al-Khateeb, A. N., Powers, J. M., Paolucci, S., Sommese, A. J., Diller, J. A., Hauenstein, J. D., and Mengers, J. D., One-dimensional slow invariant manifolds for spatially homogenous reactive systems. J. Chem. Phys. 131(2009), $042118-19$.

[19] Maas, U., and Pope, S. B., Simplifying chemical kinetics: intrinsic low-dimensional manifolds in space. Combustion and flame. 88 (3) (1992), 239 - 264. 
[20] Gorban, A. N., and Yablonsky, G. S., Extended detailed balance for systems with irreversible reactions. Chemical Engineering Science. 66(21) (2011), 5388 - 5399.

[21] Chiavazzo, E., and Karlin, I. V., Quasi-equilibrium grid algorithm: Geometric construction for model reduction. Journal of Computational Physics. 227(11) (2008), 5535 - 5560.

[22] Lebiedz, D., and Siehr, J., An optimization approach to kinetic model reduction for combustion chemistry. Flow, Turbulence and Combustion. 92(4) (2014), 885 - 902.

[23] Stahl, G., and Warnatz, J., Numerical investigation of time-dependent properties and extinction of strained methane and propane-air flamelets. Combustion and Flame. 85(3) (1991), 285 - 299. 